\title{
Development of stained polymeric nanocapsules loaded with model drugs: Use of a fluorescent poly(phenyleneethynylene)
}

\author{
Estefânia V.R. Campos a,b ${ }^{\mathrm{a}}$, Jhones L. Oliveira ${ }^{\mathrm{a}}$, Sara A. Zavala-Betancourt ${ }^{\mathrm{c}}$, \\ Antonio S. Ledezma ${ }^{c}$, Eduardo Arias ${ }^{c}$, Ivana Moggio ${ }^{c}$, Jorge Romero ${ }^{c}$, \\ Leonardo F. Fraceto ${ }^{\mathrm{a}, \mathrm{b}, *}$ \\ a Department of Environmental Engineering, State University of São Paulo, (UNESP), Sorocaba, SP, Brazil \\ ${ }^{\mathrm{b}}$ Department of Biochemistry, Institute of Biology, State University of Campinas (UNICAMP), Cidade Universitária Zeferino Vaz, Campinas, SP, Brazil

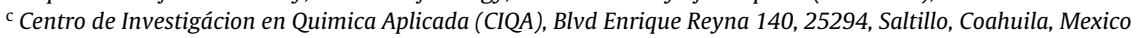

\section{A R T I C L E I N F O}

\section{Article history:}

Received 3 May 2016

Received in revised form 26 July 2016

Accepted 19 August 2016

Available online 21 August 2016

\section{Keywords:}

Stained nanocapsules

Fluorescent polymer

Polymeric nanocapsules

Environmental applications

Phenyleneethynylene polymer

\begin{abstract}
A B S T R A C T
A phenyleneethynylene polymer (here denoted pPy3E-sqS) was synthesized and characterized by UV-vis spectroscopy, fluorescence spectroscopy, and TEM, and was used for the staining of polymeric nanocapsules. The nanocapsules presented good temporal stability, without changes in shape or fluorescence, and were suitable for use in drug release systems. The mean particle size was around $430 \mathrm{~nm}$, the polydispersity index was below 0.2 , and the zeta potential was around $-13 \mathrm{mV}$. The release kinetics is one of the most important factors to consider in drug delivery systems, and here it was observed that nanocapsules containing the fluorescent polymer still maintained the ability to modulate the release of the fungicides tebuconazole and carbendazim (used as model drugs) after 4 days. Preliminary results indicated that staining with the fluorescent pPy3E-sqS polymer could be used as a valuable tool to track the behavior of polymeric systems in the environment. However, further studies will be needed to clarify the environmental behavior and possible toxicity.
\end{abstract}

(C) 2016 Elsevier B.V. All rights reserved.

\section{Introduction}

There is increasing interest in the study and application of nanomaterials in agriculture, as one of the approaches to resolving global challenges such as population growth, climate change, and the need to improve productivity while at the same time decreasing harm to the environment $[1,2]$. The application of nanotechnology in agriculture aims to reduce applications of agrochemicals, detect and treat diseases, improve the uptake of nutrients by plants, direct the release of active ingredients towards specific sites, and mitigate effects on non-target plants [2-6].

Due to the increased use of nanomaterials in various fields, including agriculture, there is a need for thorough knowledge of the interactions of these substances with both target and nontarget plants, as well as to follow their behavior in the environment, especially considering their toxicity [7-10]. To this end, the use

\footnotetext{
* Corresponding author at: Department of Environmental Engineering, State University of São Paulo, (UNESP), Sorocaba, SP, Brazil.

E-mail address: leonardo@sorocaba.unesp.br (L.F. Fraceto).
}

of stained nanocapsules has shown promise for investigating the interactions of nanomaterials with various matrices.

The use of nanocapsule systems stained with fluorescence dyes is a common approach employed to study cells and tissues in vivo and in vitro [11-13]. The advantages of using fluorescence techniques include high sensitivity, rich contrast, easy staining, and the use of standard analytical methods [14-16].

Various dyes used to stain nanomaterials exhibit high quantum yields, but in many cases, problems of cytotoxicity have limited their broad application in biological studies [14-16]. It is therefore important to develop and evaluate new materials with potential for use in future applications in areas including agriculture, environment, and bio-medicine.

The chain structures of conjugated polymers show alternation of single and double or triple bonds, creating extended delocalization of electrons and consequently the generation of electronic properties [17-19]. The excitation energies of these $\pi$-conjugated materials are usually in the visible range, making them optically active [20].

In this work, we report the preparation and characterization of polymeric nanocapsules consisting of polycaprolactone and 


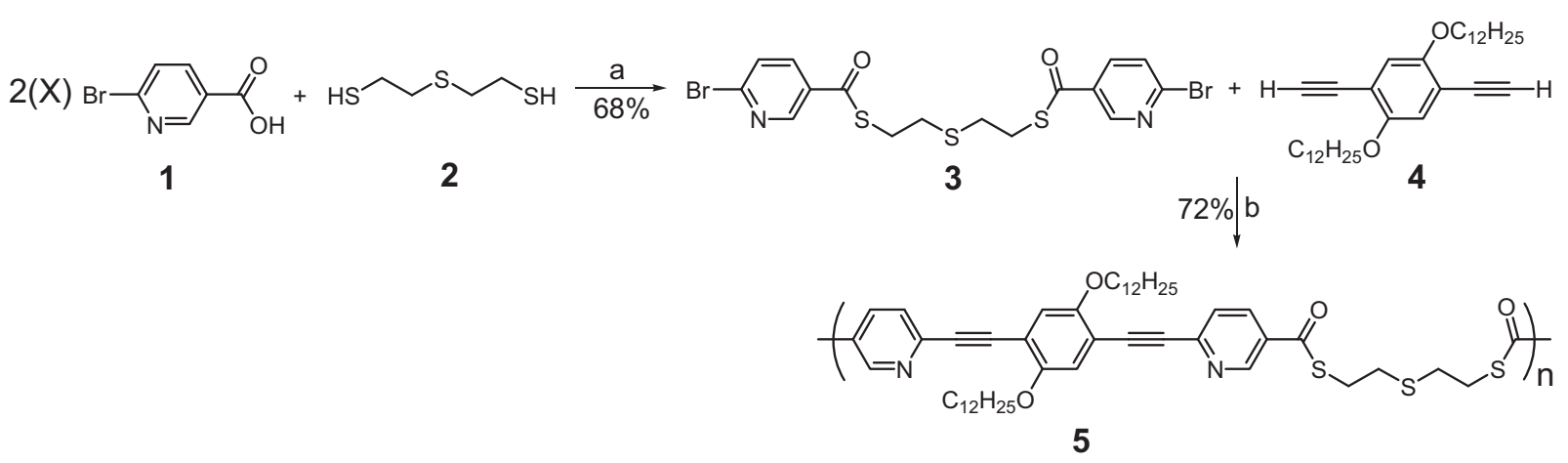

Scheme 1. Reagents and conditions: (a) DCC/DMAP, $\mathrm{CH}_{2} \mathrm{Cl}_{2}, 0$ to r.t. ${ }^{\circ} \mathrm{C}, 15 \mathrm{~h}$; (b) $\left[\left(\mathrm{C}_{6} \mathrm{H}_{5}\right)_{3} \mathrm{P}\right]_{2} \mathrm{PdCl}_{2}(2.5 \mathrm{~mol} \%), \mathrm{CuI}(1.5 \mathrm{~mol} \%), \mathrm{Et}{ }_{3} \mathrm{~N}, 80{ }^{\circ} \mathrm{C}, 36 \mathrm{~h}$.

stained with a fluorescent poly(phenyleneethynylene) sequenced with flexible thioester-diethyldisulfide chains. This system, hereafter denoted pPy3E-sqS, has the potential to enable the detection of nanocapsules by UV/Vis and fluorescent spectroscopy, as well as by laser scanning confocal microscopy (LSCM). The feasibility of different formulations of nanocapsules, unstained or stained with pPy3E-sqS, were evaluated as carrier systems by loading them with tebuconazole and carbendazim. These compounds, which are fungicides, were used here as model drugs. The physicochemical stabilities and the release profiles of the loaded and unloaded fluorescent nanocapsules were assessed as a function of time. This study opens perspectives for monitoring the interactions of polymeric nanocapsules with target and non-target organisms in agriculture, such as their penetration into leaves, interaction with the soil matrix, and diffusion to water resources, in order to understand the ways in which nanocapsules interact with these complex systems. In addition, nanocapsules stained with pPy3E-sqS could be employed to evaluate the toxicity in soil (such as towards worms Caenorhabditis elegans), plants, and insects.

\section{Materials and methods}

\subsection{Materials}

The following chemical products were obtained from Aldrich and were used without further purification: polycaprolactone (PCL), poly(vinyl alcohol) (PVA), copper(I) iodide, dichlorobis(triphenylphosphine) palladium(II), 6bromopyridine-3-carboxylic acid, 2-mercaptoethylsulfide, sodium diethyldithiocarbamate, dicyclohexylcarbodiimide (DCC), and dimethylaminopyridine (DMAP). $\mathrm{CH}_{2} \mathrm{Cl}_{2}, \mathrm{CHCl}_{3}$, hexane, and methanol were purchased from Aldrich and JT Baker. THF and triethylamine were distilled from sodium/benzophenone complex and $\mathrm{KOH}$, respectively.

\subsection{Synthesis of pPy3E-sqS}

S,S'-2,2'-thiobis(ethane-2,1-diyl) carbothioate) (compound $\mathbf{3}$ in Scheme 1)

A round bottom flask fitted with a stirrer, condenser, and calcium chloride guard tube was loaded with 6-bromopyridine3-carboxylic acid (3.3 g, $16.33 \mathrm{mmol}$ ) (compound 1 in Scheme 1), 2-mercaptoethylsulfide $(0.96 \mathrm{~mL}, 7.42 \mathrm{mmol}$ ) (compound 2 in Scheme 1), and $80 \mathrm{~mL}$ of $\mathrm{CH}_{2} \mathrm{Cl}_{2}$ (methanol free). The solution was cooled to $\sim 0{ }^{\circ} \mathrm{C}$, followed by dropwise addition of DCC $(3.306 \mathrm{~g}$, $16.0 \mathrm{mmol}$ ) and DMAP (55 mg, $0.445 \mathrm{mmol}$ ) dissolved in $10 \mathrm{~mL}$ of $\mathrm{CH}_{2} \mathrm{Cl}_{2}$. The mixture was then heated at $50^{\circ} \mathrm{C}$ overnight. The urea formed was filtered off and the filtrate was washed with a $0.5 \mathrm{M} \mathrm{HCl}$ solution. The mixture was transferred to a separating funnel and the organic layer was extracted with $\mathrm{CH}_{2} \mathrm{Cl}_{2}$ and dried with $\mathrm{MgSO}_{4}$. After filtering off the drying agent, the solvent was removed under vacuum and the crude product was chromatographed through a silica gel column using $\mathrm{CH}_{2} \mathrm{Cl}_{2}$ as eluent, resulting in a $68 \%$ yield of a white powder with the following characteristics: $\mathrm{mp} 141-143^{\circ} \mathrm{C}$; ${ }^{1} \mathrm{H} \mathrm{NMR}\left(\mathrm{CDCl}_{3}, 300 \mathrm{MHz}\right) \delta(\mathrm{ppm}): 8.8$ (s, 2H, H-Ar), 8.0 (d, 2H, H$\mathrm{Ar}), 7.62$ (d, 2H, H-Ar), 3.35 (t, $\left.4 \mathrm{H},-\mathrm{COS}-\mathrm{CH}_{2}\right), 2.9\left(\mathrm{t}, 4 \mathrm{H},-\mathrm{CH}_{2}-\mathrm{S}-\right.$ ); ${ }^{13} \mathrm{C} \mathrm{NMR}\left(\mathrm{CDCl}_{3}, 75 \mathrm{MHz}\right) \delta(\mathrm{ppm}): 189.06(\mathrm{C}=\mathrm{O}), 148.93(\mathrm{~N}-\mathrm{C} 2)$, 147.23 (C6-Br), 136.61 (C4), 131.57 (C3), 128.30 (C5), 31.59 (-CO$\left.\mathrm{S}-\mathrm{CH}_{2}-\right), 29.29$ (- $\left.\mathrm{CH}_{2}-\mathrm{S}-\right)$.

Degassed $\mathrm{Et}_{3} \mathrm{~N}(40 \mathrm{~mL})$ was added through a dry cannula, under a nitrogen atmosphere, to a two-neck round bottom flask containing compound 3 (220 mg, $0.42 \mathrm{mmol}),\left[\left(\mathrm{C}_{6} \mathrm{H}_{5}\right)_{3} \mathrm{P}\right]_{2} \mathrm{PdCl}_{2}$ $(7.8 \mathrm{mg}, 0.11 \mathrm{mmol})$, and $\mathrm{CuI}$ (ca. $1.0 \mathrm{mg})$. The mixture was heated at $60^{\circ} \mathrm{C}$ for $15 \mathrm{~min}$. Subsequently, 1,4-diethynyl-2,5bis(dodecanoxy)benzene (compound 4) (189 mg, $0.38 \mathrm{mmol}$ ) dissolved in dry and degassed $\mathrm{Et}_{3} \mathrm{~N}(5 \mathrm{~mL})$ was added using a syringe, followed by stirring for $36 \mathrm{~h}$ at $80^{\circ} \mathrm{C}$. After cooling, the mixture was filtered to eliminate the ammonium salt, washed with THF, and the organic phase was evaporated to a minimal volume prior to precipitation with methanol $(50 \mathrm{~mL})$ containing sodium diethyldithiocarbamate ${ }^{21}(15 \mathrm{mg})$, followed by centrifugation. The pasty residue was recovered into $4 \mathrm{~mL}$ of $\mathrm{CHCl}_{3}$ and precipitated twice in clean methanol. Finally, the product was passed through a preparative SEC column (Bio-Beads SX1, Bio-Rad) using toluene as eluent. It was then concentrated, dissolved in $10.5 \mathrm{~mL}$ of $\mathrm{CHCl}_{3}$, and refrigerated until used. The yield was $72 \%, \lambda_{\mathrm{abs}}(\varepsilon)=408 \mathrm{~nm}$ $\left(79.98 \mathrm{~g} \mathrm{~L}^{-1} \mathrm{~cm}^{-1}\right)$, and $\lambda_{\text {fluo }}(\phi)=480 \mathrm{~nm}(0.30 \pm 0.02)$.

\subsection{Characterization of $p P y 3 E-s q S$}

A JEOL Eclipse spectrometer was used to acquire ${ }^{1} \mathrm{H}$ NMR (300 MHz) and ${ }^{13} \mathrm{C}$ NMR $(75.4 \mathrm{MHz})$ spectra at room temperature, using $\mathrm{CDCl}_{3}$ as the solvent and internal standard. The molecular weight of the polymer was determined by GPC, using an Agilent Technologies 1200 series HPLC (Model 61316A) with PS standards, refractive index detector, and toluene as the mobile phase at $45^{\circ} \mathrm{C}$ and $1 \mathrm{~mL} \mathrm{~min}^{-1}$. For the photophysical characterization of the polymer, spectroscopic grade chloroform (Sigma-Aldrich) was used. UV-vis spectra were acquired with an Agilent 8453 spectrophotometer. Fluorescence emission and excitation spectra were obtained using a Perkin Elmer LS50 B spectrofluorimeter operated at an excitation wavelength of $394 \mathrm{~nm}$. The temperature was controlled at $25.0 \pm 0.3^{\circ} \mathrm{C}$ by means of a recirculating water bath. The fluorescence quantum yield was determined according to the literature, using $0.1 \mathrm{M} \mathrm{H}_{2} \mathrm{SO}_{4}$ quinine sulfate $(\phi=0.54)[22]$ as a standard. Three different samples with optical density at $394 \mathrm{~nm}$ 
lower than 0.1 were analyzed, and the values were averaged. Lifetimes were determined with a flash photolysis spectrometer (LP920 KS, Edinburgh Instruments). A 0.01\% suspension of Ludox AS40 (Aldrich) in ultrapure water was used for the prompt signal. The data were fitted using the instrument software.

The average lifetime $(\tau)$ was calculated from the lifetimes obtained $\left(\tau_{i}\right)$, using the following equation:

$\tau=\Sigma_{1}^{n} f_{i} \tau_{i}$

where $f_{i}$ is the fractional fluorescence contribution of each lifetime $\tau_{i}$, given by:

$f i=\frac{\alpha_{i} \tau_{i}}{\Sigma_{1}^{n} \alpha_{i} \tau_{i}}$

and $\alpha$ is the pre-exponential coefficient obtained from the fit.

\subsection{Preparation of the polymeric nanocapsules}

The emulsion/solvent evaporation technique was employed to prepare the polymeric nanocapsules [24]. Briefly, a known amount of the pPy3E-sqS fluorescent polymer was dissolved in $10 \mathrm{~mL}$ of acetone. Simultaneously, another solution was prepared containing $400 \mathrm{mg}$ of PCL dissolved in $20 \mathrm{~mL}$ of chloroform. These solutions were emulsified by ultrasonication for $1 \mathrm{~min}$ at $90 \mathrm{~W}$. This primary emulsion was added to an aqueous solution $(50 \mathrm{~mL})$ containing $150 \mathrm{mg}$ of PVA, and the resultant solution was ultrasonicated for $8 \mathrm{~min}$ to form a double emulsion. Finally, the organic solvent was removed with the aid of a rotary evaporator and the emulsion was concentrated to a final volume of $10 \mathrm{~mL}$. Taking into account the mass of PCL polymer used in the preparation of the nanocapsules, different concentrations of the fluorescent $\mathrm{pPy} 3 \mathrm{E}-\mathrm{sq} \mathrm{S}$ polymer $(0.5$, $1,2,3$, and $4 \% \mathrm{w} / \mathrm{w}$ ) were added to the formulations in order to identify the concentration that resulted in the greatest coverage of the nanocapsules. Nanocapsules without fluorescent polymer were prepared as a control.

\subsection{Polymeric nanocapsules characterization}

Characterization of the nanocapsules was performed using fluorescence spectra obtained for the samples immediately after preparation and following storage under refrigeration for different time intervals. Zeta potential measurements were carried out using a ZetaSizer Nano ZS90 analyzer (Malvern Instruments), at a fixed angle of $90^{\circ}$ and temperature of $25^{\circ} \mathrm{C}$. NTA analyses employed a laser with a wavelength of $532 \mathrm{~nm}$ (green), a CMOS camera, and NanoSight v. 2.3 software [25]. Electron microscopy was performed using an FEI Titan $300 \mathrm{kV}$ field emission gun microscope equipped with an S-TWIN symmetrical condenser-objective lens with a spherical aberration coefficient (Cs) of $1.25 \mathrm{~mm}$. The images were acquired with a CCD camera. A Zeiss-LEO microscope (Model 906) was employed to investigate the morphology of the nanocapsules. Laser scanning microscopy images were acquired with a Zeiss Pascal 5 instrument, with excitation using an argon $458 \mathrm{~nm}$ laser. A two-channel configuration was chosen in order to obtain fluorescence and reflection images. In the fluorescence image, only the nanocapsules coated with the polymer could be seen, while the reflection image showed the entire sample. The color of the fluorescence was selected from the palette of the software and was therefore not a direct visualization of the emission. The samples were prepared using polymer concentrations of $0.5,1,2,3$, and $4 \%$. A $10 \mu \mathrm{L}$ aliquot of each suspension was diluted in $10 \mathrm{~mL}$ of water, and $0.1 \mathrm{~mL}$ of this preparation was dropped onto a microscopic slide and left to dry for one day at room temperature, prior to the analysis.

\subsection{Release experiments}

Drug release kinetic assays were performed in order to determine whether addition of the fluorescent pPy3E-sqS influenced the profile of release from the nanocapsules [26]. For this purpose, carbendazim and tebuconazole were used as model drugs, in order to enable comparison with previously published results [27]. The release assays were performed under dilution sink conditions, with constant stirring. Appropriate amounts of the formulations were diluted in the central acceptor compartment (using deionized water). Aliquots were collected and transferred to Falcon tubes at intervals of 15,30 , and 60 min during the first eight hours, after which two aliquots were obtained every $24 \mathrm{~h}$, up to $96 \mathrm{~h}$. The samples were centrifuged in order to sediment out the nanocapsules, and the concentrations of the compounds were determined by HPLC.

\section{Results and discussion}

\subsection{Polymer synthesis and characterization}

The semi-rigid polymer (compound 5), here denoted pPy3EsqS, was synthesized according to the chemical route given in Scheme 1 . The pathway essentially consisted of two reactions: i) thioesterification of 6-bromopyridine-3-carboxylic acid (1) with 2mercaptoethylsulfide (2) under the $N, N$ '-dicyclohexylcarbodiimide (DCC)/DMAP-mediated esterification conditions, in $\mathrm{CH}_{2} \mathrm{Cl}_{2}$, to give $\mathrm{S}, \mathrm{S}^{\prime}-2,2^{\prime}$-thiobis(ethane-2,1-diyl) bis(6-bromopyridine-3carbothioate) (3), with a yield of $68 \%$; ii) the Pd/CuI cross-coupling reaction between the corresponding dibromide terminated monomer (3) and 1,4-diethynyl-2,5-bis(dodecanoxy)benzene (8) [15]. The use of a co-solvent was avoided, because it would drastically increase the molecular weight, which usually gives rise to insoluble aggregates.

The polymer was submitted to a metal decomplexation process by precipitation, once with a methanol/sodium dithiocarbamate solution, and twice with clean methanol. The shortest oligomers were eliminated using a preparative SEC column (Bio-Beads SX1, Biorad), with toluene as eluent. The proton resonance peaks of pPy3E-sqS were broader, compared to those of the corresponding monomers; the acetylenic proton disappeared, while the protons near the bromine could be identified, suggesting that the molecular chains were mainly terminated by bromines (Fig. 1S). The polymer (5) was soluble in chloroform, THF, and toluene. It was partially soluble in dipolar solvents such as dimethylformamide and dimethylsulfoxide, and was precipitated in polar solvents such as methanol and ethanol. The average molecular weight, calculated using PS standards, was $26504 \mathrm{~g} / \mathrm{mol}$, and the polydispersity index was 2.99 (typical of polycondensed conjugated polymers). The polymer presented conjugated and fluorescent phenyleneethynylene units alternating with flexible sequences containing sulfur atoms, imparting a partially amphiphilic character that improved its solubility in common organic solvents, preventing chain aggregation due to $\pi-\pi$ electronic interactions and allowing interaction with poly(caprolactone).

Fig. 1A shows the UV-vis, excitation, and fluorescence spectra in $\mathrm{CHCl}_{3}$. The polymer exhibited a main absorption peak at $403 \mathrm{~nm}$, which could be attributed to HOMO-LUMO electronic transitions, and a higher energy peak at $324 \mathrm{~nm}$, probably due to the HOMO1/LUMO transition associated with the alkoxy substitution in the phenyleneethynylene. The emission spectrum presented a broad band with maximum at $480 \mathrm{~nm}$, at any excitation wavelength, as shown in Fig. 1B. The excitation spectrum corresponded to the absorption spectrum, as can be seen in Fig. 1A. These two latter features indicated that the emission was always generated by the same 

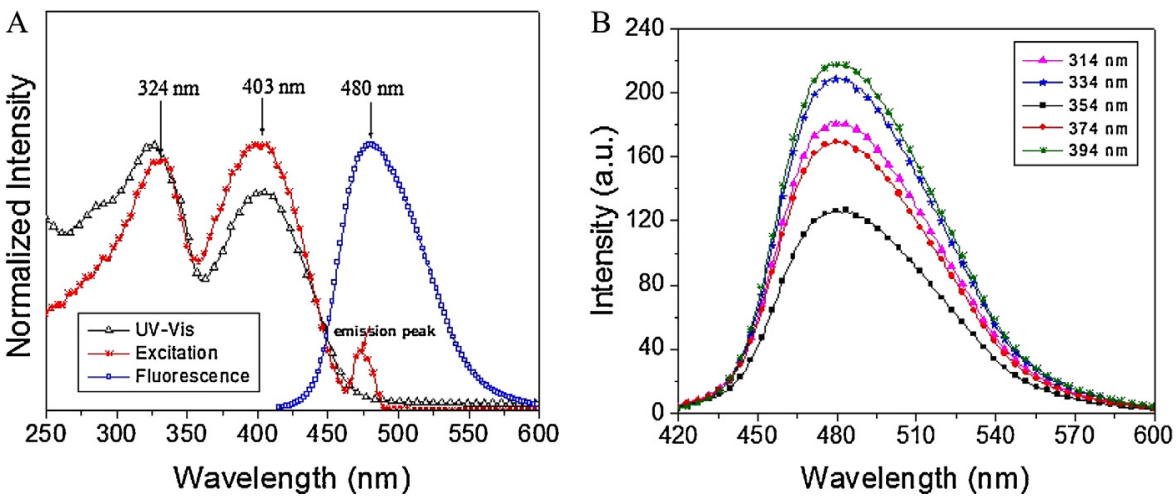

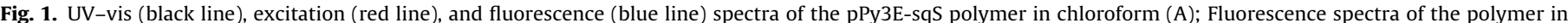

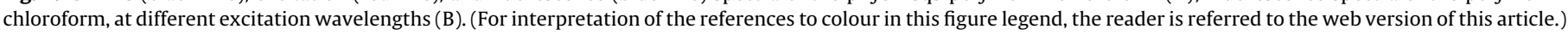

electronic transition and that the two absorption bands were associated with only one chromophore. The fluorescence quantum yield $(\phi=0.30)$ was on the same order of magnitude reported for other poly(phenyleneethynylene)s, and somewhat lower than found for a PPE with similar sequence.

The fluorescence decay showed a bi-exponential fit (Fig. S2, Supporting information), with an average lifetime of $1.69 \mathrm{~ns}$. From the values of quantum yield and lifetime, a non-radiative constant $\left(\mathrm{k}_{\mathrm{nr}}\right)$ of $0.41 \mathrm{~ns}$ and a radiative constant $\left(\mathrm{k}_{\mathrm{rad}}\right)$ of $0.18 \mathrm{~ns}$ were obtained. Despite the high absorption coefficient $\left(\varepsilon=79.98 \mathrm{~g} \mathrm{~L}^{-1} \mathrm{~cm}^{-1}\right)$, the fact that $\mathrm{k}_{\mathrm{nr}}$ was greater than $\mathrm{k}_{\text {rad }}$ was in agreement with the fluorescence quantum yield and revealed that de-excitation processes competed with fluorescence. As the Stokes's shift $\left(\Delta \mathrm{n}=4014 \mathrm{~cm}^{-1}\right)$ was quite large, internal conversion was probably one of the possible de-excitation mechanisms. However, the possible existence of intersystem crossing could not be excluded, especially as bromine terminal groups were present (as indicated by the NMR spectra). Overall, a quantum yield of $30 \%$, together with the partially amphiphilic character of the polymer, indicated that pPy3E-sqS should be a good candidate for use as a fluorescent dye.

\subsection{Staining and characterization of the nanocapsules}

Different weight percentages of pPy3E-sqS were added to the poly(epsilon-caprolactone) nanocapsules and the samples were first analyzed by LSCM microscopy. As an example, Fig. 2 shows the fluorescence and reflection images obtained for nanocapsules stained with $3 \%$ of the fluorescent pPy3E-sqS. The images for the other polymer percentages are provided in the Supporting information (Fig. S3).

The poly(epsilon-caprolactone) nanocapsules did not emit under the excitation conditions used, as can be observed for the image corresponding to $0 \%$ of polymer. The nanocapsules appeared to be quasi-spherical, although some particles coalesced due to the drying process that was necessary for the LSCM analyses. At $0.5 \%$, practically no emission could be seen, while fluorescent nanocapsules began to appear at a polymer concentration of $1 \%$. The quantity of fluorescent spots increased with the polymer concentration, and at $3 \%$ all of the nanocapsules present in the reflection image could also be seen in the fluorescence channel. At $4 \%$, there was a clear decrease in fluorescence intensity, indicative of reabsorption effects. Fluorescence spectra of the different suspensions were acquired separately with a fluorimeter.

Based on the LSCM observations, the spectrum for the $0.5 \%$ suspension was not included in Fig. 3, which illustrates the fluorescence spectra of the particles with $1,2,3$, and $4 \%$ of polymer dissolved in distilled water. These spectra showed a red shift, relative to the spectrum of the polymer in chloroform (Fig. 1B), which could be explained by the solid-state effects reported for conjugated polymers, in particular if the polymer is considered to interact with the capsule as a thin layer. There was also a red shift as the percentage of polymer increased, which could again be attributed to solid-state effects, with increased thickness of the polymer layer coating resulting in decreased intensity. Despite the fact that UV-vis spectra could not be recorded, due to the turbidity of the suspensions, the red shift suggested that the thickness increased, so an increase in the absorption was expected. Hence,
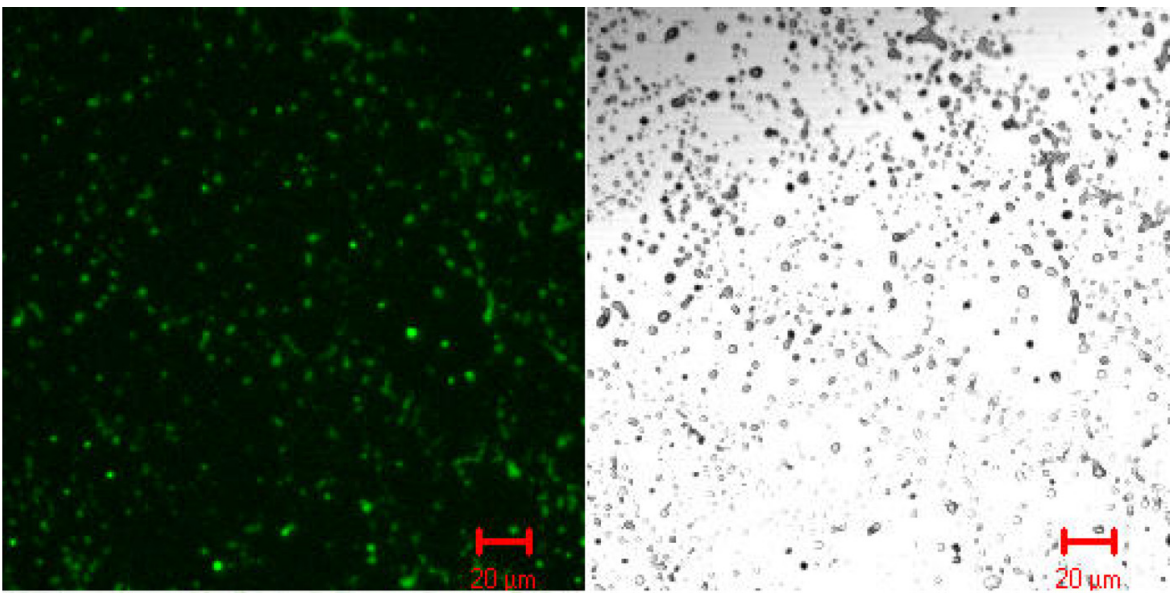

Fig. 2. LSCM fluorescence (left) and reflection (right) images of nanocapsules coated with $3 \%$ of the fluorescent pPy3E-sqS. 


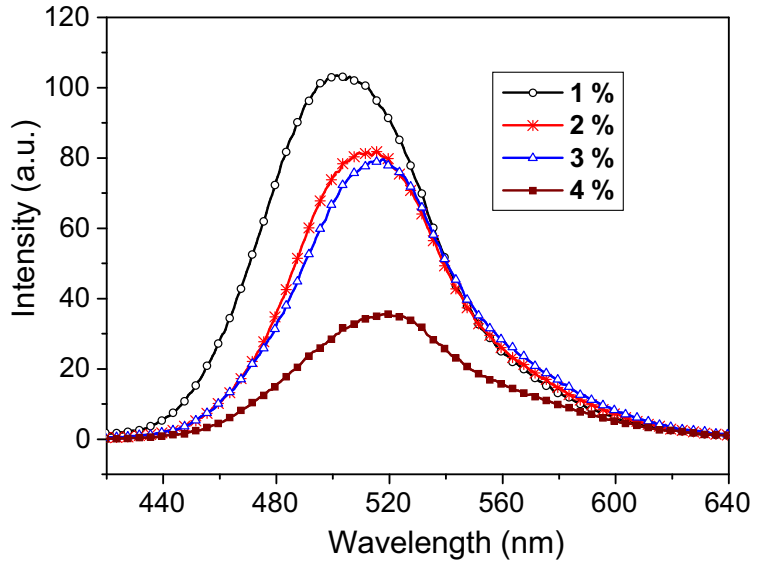

Fig. 3. Fluorescence spectra of the nanocapsules stained using different polymer concentrations.

the fluorescence quenching was probably due to self-absorption. In terms of fluorescence intensity, the best results were obtained using $1 \%$ polymer. However, the LSCM results indicated that complete coating of the particles was only achieved with concentrations of $3 \%$ and above. As the intensity was dramatically quenched at $4 \%$, a concentration of $3 \%$ was taken as the optimum, considering both the spectroscopic and microscopic characterizations.

In order to obtain further information concerning the staining process, the pPy3E-sqS polymer and the stained nanocapsules were analyzed using HRTEM. Fig. 4a shows a planar view of a film of pPy3E-sqS with a calculated periodicity of $0.25 \pm 0.02 \mathrm{~nm}$. Selected area electron diffraction (SAED) analysis (Fig. 4a insert) showed a reflection at $d=0.25 \mathrm{~nm}$. These distances correspond to the separation between conjugated chains, where the dodecanoxy side chains are lengthened perpendicularly to the film surface, as indicated in Fig. $4 \mathrm{~b}$, suggesting that strong $\pi-\pi$ interaction between conjugated segments predominated in this polymer, as usually found for phenyleneethynylene macromolecules [28].

In order to investigate the effect of staining with the polymer on the nanocapsules, and to determine whether $3 \%$ of fluorescent polymer was sufficient to provide full coverage, a drop of stained nanocapsules was observed by TEM (Fig. 4c), and a section of one nanocapsule was submitted to HRTEM analysis (Fig. 4d). Three regions could be clearly identified: i) a region with periodicity of $0.25 \mathrm{~nm}$, similar to that of the conjugated polymer backbones assembled parallel to the surface, as previously discussed; ii) a region where the polymer backbones lay flat on the surface; and iii) amorphous regions associated with the poly(caprolactone) nanocapsule wall (shown with an arrow), indicating that the nanocapsules were not totally covered by the pPy3E-sqS. The illustration in Fig. 4e provides a visual overview of the staining process. Increasing the content of polymer led to a tendency of the polymer on the nanocapsule surface to self-assemble, hence increasing the thickness, which was consistent with the greater quenching of fluorescence at higher polymer concentrations, described previously. In other words, the self-assembling of the polymer to form blocks of molecules was stronger than its interaction with the nanocapsule surface. This hypothesis will be confirmed using a similar polymer, but with glycol side chains (currently under synthesis).

\subsection{Colloidal stability of the stained polymeric nanocapsules}

Before investigation of the release profiles of the test compounds from the nanocapsules coated with pPy3E-sqS, the particle stability was evaluated by measuring the mean diameter, polydispersity index, zeta potential, and fluorescence intensity, as a function of time (up to 90 days). The mean diameter obtained by DLS for the nanocapsules without pPy3E-sqS (Fig. 5a, black columns) was $249.3 \pm 3.4 \mathrm{~nm}$ immediately after preparation and remained without significant changes during the storage period, with a value of $245.6 \pm 5.1 \mathrm{~nm}$ after 90 days. The polydispersity index (Fig. 5a, black line) of the nanocapsules remained below 0.2 throughout the storage period, indicating good homogeneity of the nanocapsule size distribution. The nanocapsules with pPy3E-sqS (Fig. 5a, red column) showed a greater mean diameter $(428.5 \pm 4.1 \mathrm{~nm})$, compared to the control nanocapsules, and maintained this size distribution range during the 90 days of storage, without any significant changes. This increase in the mean diameter was indicative of incorporation of the fluorescent polymer in the polymeric network of the nanocapsules, as previously discussed for the HRTEM images. The polydispersity index (Fig. 5a, red line) showed a value of $0.29 \pm 0.05$ immediately after preparation, then decreased to 0.2 after 15 days and subsequently remained constant up to 90 days of storage

Measurement of the surface charge of nanocapsules, known as the zeta potential, provides an indication of the stability of colloidal systems; higher values (positive or negative) are indicative of greater stability. The initial zeta potentials (Fig. 5b) were $-13.1 \pm 1.3 \mathrm{mV}$ and $-6.9 \pm 1 \mathrm{mV}$ for nanocapsules with and without the fluorescent polymer, respectively, and the values increased after 90 days of storage. However, the surfactant used during the synthesis of the nanocapsules was poly(vinyl alcohol), which is known to stabilize colloidal systems by steric hindrance, so electrostatic stabilization was not the main mechanism responsible for stabilization of the formulations $[21,22,23,29]$.

The mean diameters of the nanocapsules with and without pPy3E-sqS were measured using nanoparticle tracking analysis (NTA), as a function of storage time. Unlike the PSC technique, which determines the size distribution of a set of nanoparticles, NTA analyzes each particle individually. In addition to the size distribution, this technique is able to determine the concentration of nanocapsules present in the formulation (Fig. 6). For the nanocapsules without pPy3E-sqS, the same mean diameters were obtained by both techniques (DLS and NTA). The concentration of the control nanocapsules was around $(5.60 \pm 0.65) \times 10^{12}$ particles $/ \mathrm{mL}$, and remained almost constant during 90 days. There was no formation of aggregates (which would have been observed if the concentration of the nanocapsules decreased over time). Unlike the control nanocapsules, in the case of the nanocapsules with the fluorescent polymer, a smaller diameter was obtained using NTA $(286.6 \pm 5.40 \mathrm{~nm})$, compared to DLS $(428.5 \pm 4.11 \mathrm{~nm})$. Although both techniques measure Brownian motion, there is a difference in the nature of the analysis. NTA identifies and tracks the movement of the particles on a particle-by-particle basis, while DLS does not observe the particle, but instead uses the dispersion in light intensity caused by constructive and destructive interferences, hence explaining the difference between the two techniques in terms of particle size. The nanocapsules with pPy3E-sqS showed a concentration of $(7.05 \pm 0.23) \times 10^{12}$ particles $/ \mathrm{mL}$.

Finally, the fluorescence of the pPy3E-sqS in the formulation was characterized using spectroscopic analysis. The results (Fig. 7) showed that there was a slight decrease in fluorescence intensity for the nanocapsules with $3 \%$ of pPy3E-sqS, with an approximately $20 \%$ reduction in intensity after 90 days of storage.

\subsection{Release kinetics of model drugs}

Two model molecules (carbendazim and tebuconazole) were employed to evaluate whether the presence of pPy3E-sqS altered the release kinetics, compared to the values reported previously for nanocapsules without pPy3E-sqS [27]. 


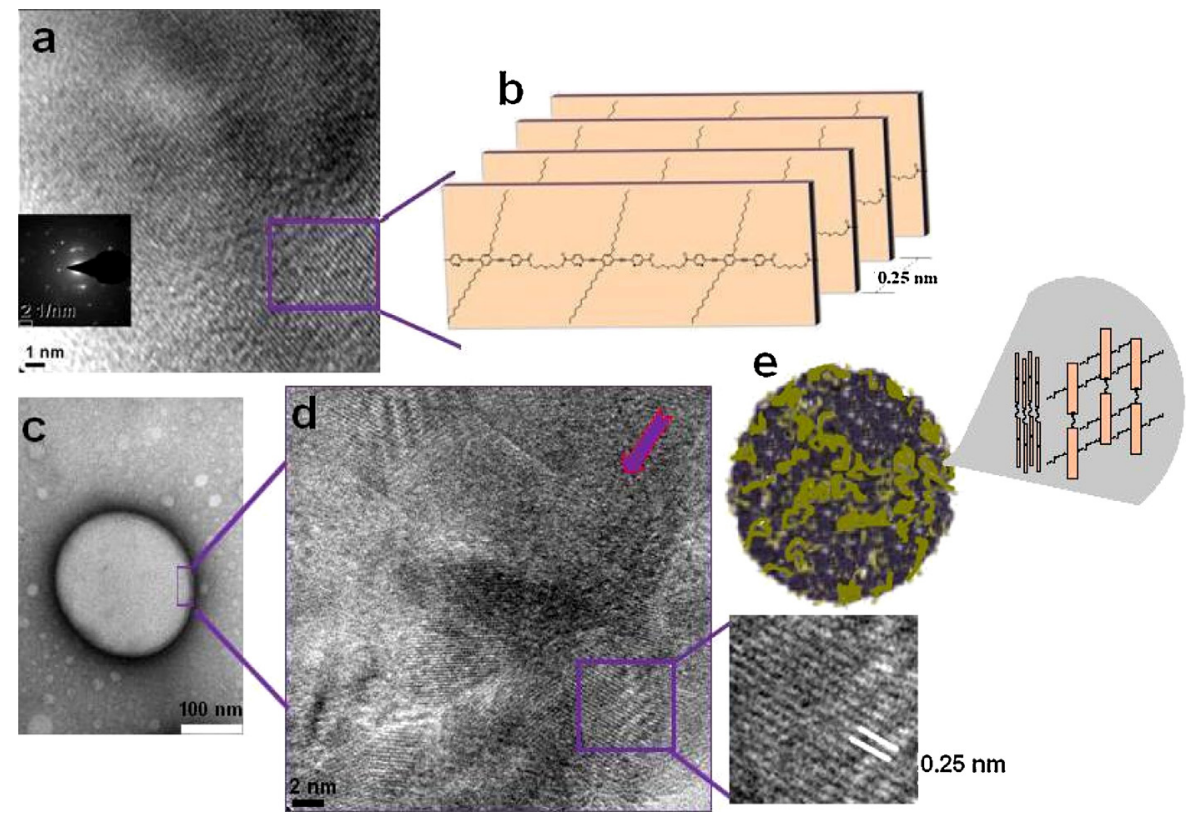

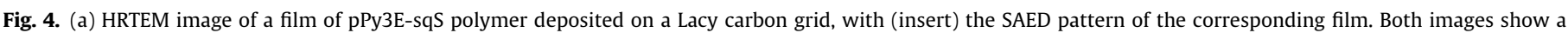

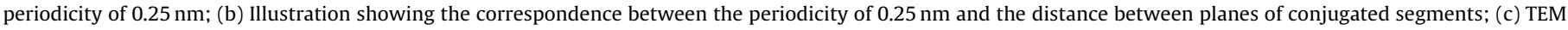

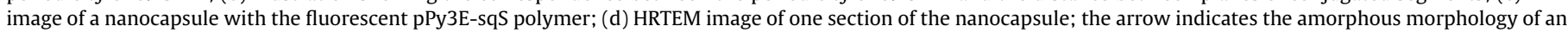

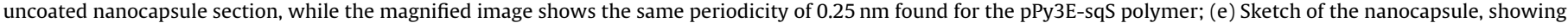
self-assembly of the pPy3E-sqS polymer in two configurations.
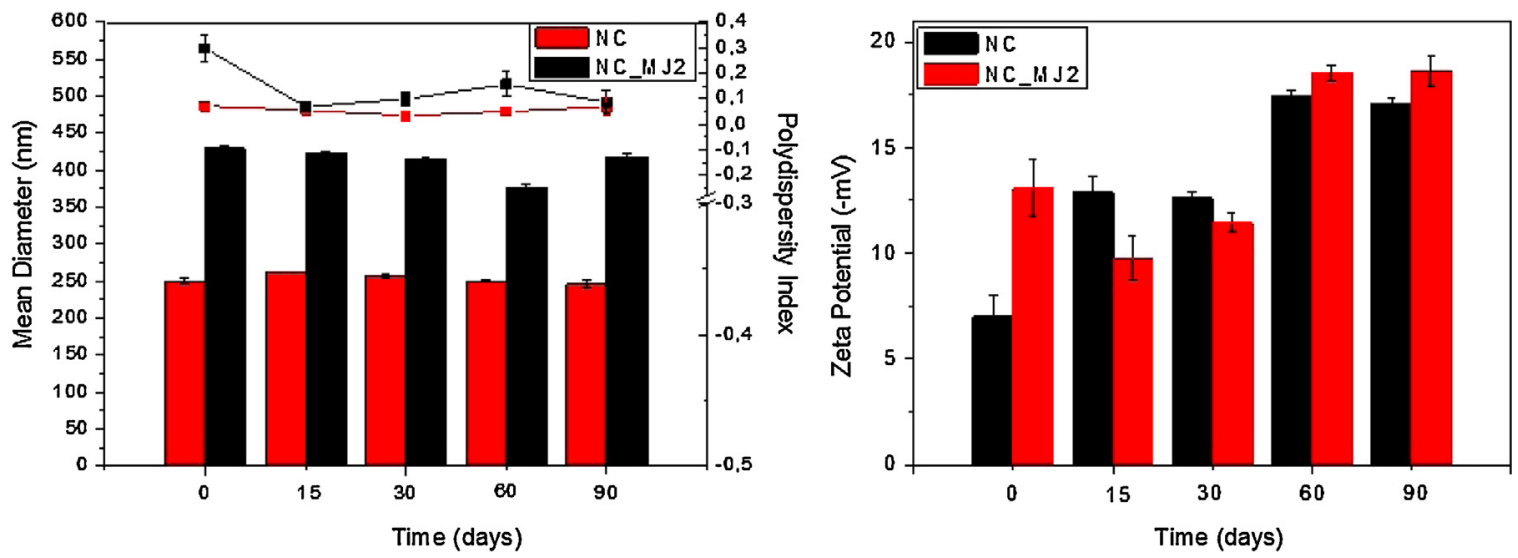

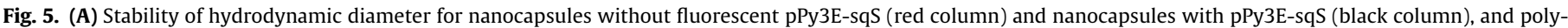

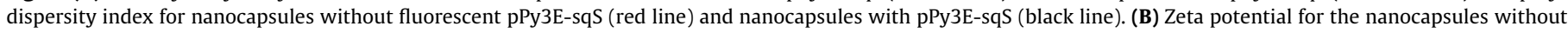

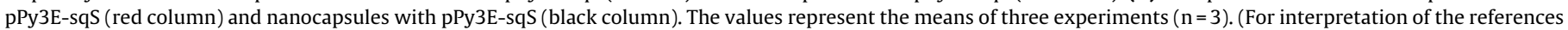
to colour in this figure legend, the reader is referred to the web version of this article.)
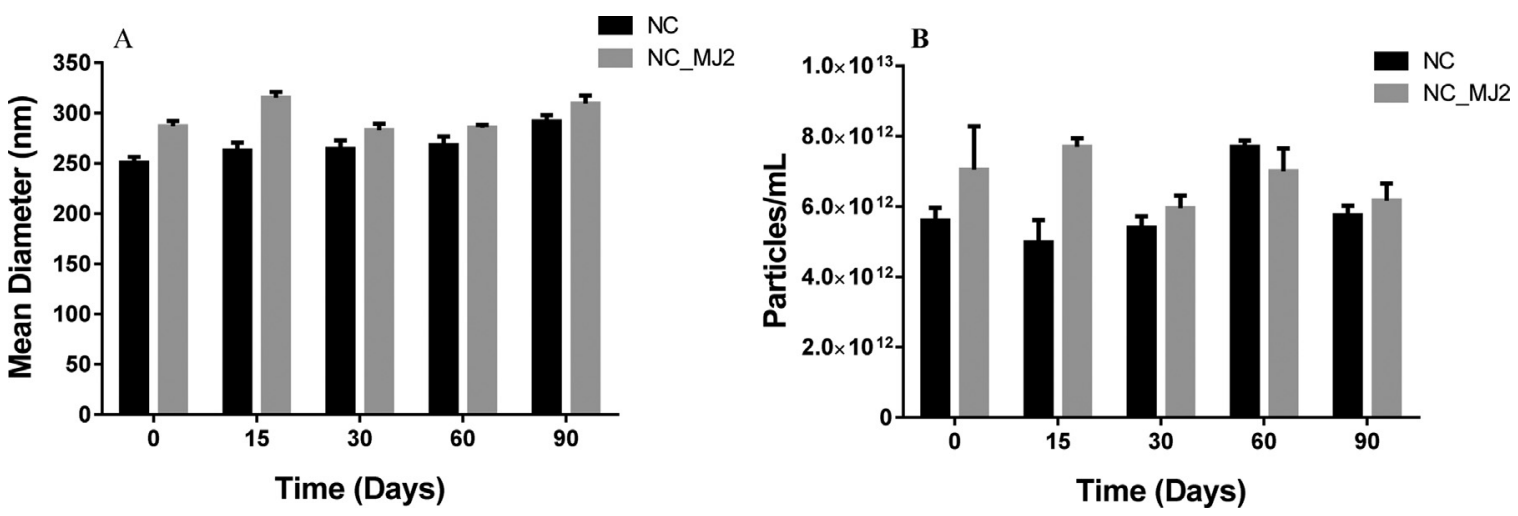

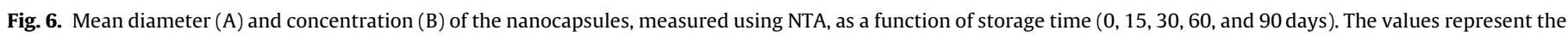
means of three experiments $(n=3)$. 


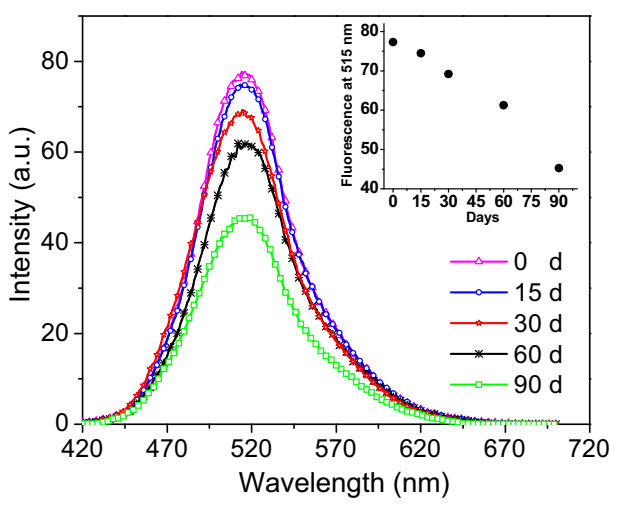

Fig. 7. Time-dependent fluorescence intensity of the nanocapsules stained with $3 \%$ of fluorescent pPy3E-sqS polymer. Insert: fluorescence intensity at $515 \mathrm{~nm}$, plotted against time.

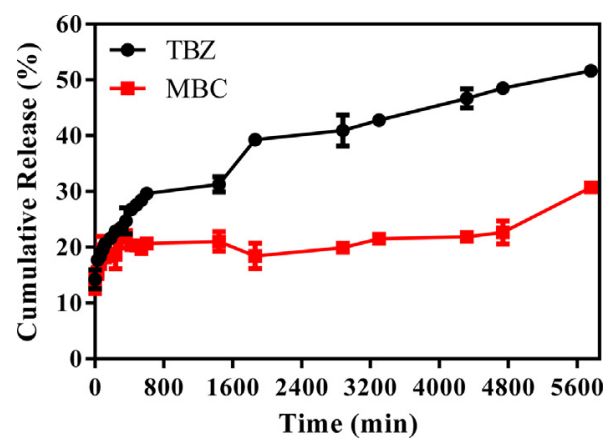

Fig. 8. Cumulative release of carbendazim and tebuconazole from nanocapsules containing the fluorescent pPy3E-sqS polymer. The values represent the means of three experiments $(n=3)$.

The results (Fig. 8) showed that for both compounds, there was a rapid release from the interior of the nanocapsules during the first $600 \mathrm{~min}$, followed by sustained release. After $5760 \mathrm{~min}$, around 30\% of the carbendazim incorporated in the nanocapsules was released, while around $50 \%$ of the tebuconazole was released over the same period. A possible explanation for the $\mathrm{MBC}$ release profile is that from 0 to $400 \mathrm{~min}$, the $\mathrm{MBC}$ molecules that were associated more superficially with the nanocapsules were released. Between 400 and $4800 \mathrm{~min}$, the cumulative release remained constant, while after $4800 \mathrm{~min}$, the MBC molecules that had been inserted into the nanocapsule cores began to be released to the environment. Hence, there was slow release of the encapsulated MBC. These results were in agreement with those obtained for nanocapsules without pPy3E-sqS, where $20 \%$ of carbendazim and $46 \%$ of tebuconazole were released after $5760 \mathrm{~min}$ [27]. This indicated that the conjugated polymer did not affect the process of release from the nanocapsules.

\section{Conclusions and perspectives}

This work describes the synthesis and characterization of a fluorescent conjugated polymer and its incorporation on the surface of polymeric nanocapsules, with the aim of developing an optical detection system for the tracking and monitoring of nanoparticles in the environment. Considering that incorporation of the conjugated polymer might cause destabilization, the system was evaluated using physicochemical characterization and investigation of the kinetics of release of active agents from the stained nanocapsules.

A semi-rigid rod-like poly(phenyleneethynylene) sequenced with flexible thioester-diethyldisulfide chains, denoted pPy3E-sqS, was synthesized by the Sonogashira cross-coupling reaction. In chloroform, the pPy3E-sqS polymer emitted high energy light in the blue region at $480 \mathrm{~nm}$, with a high quantum yield, making it a good candidate for staining nanocapsules.

Analyses using LSCM and HRTEM microscopy confirmed that the fluorescent polymer (pPy3E-sqS) interacted with the polymeric wall of the nanocapsules. The stained nanocapsules showed a mean diameter of around $428.5 \pm 4.1 \mathrm{~nm}$ and remained stable over a period of 90 days. NTA and TEM analysis showed that the nanocapsules were spherical, with an average concentration of $(7.05 \pm 0.23) \times 10^{12}$ particles $/ \mathrm{mL}$. The release profiles of the model drugs were not affected by the presence of the fluorescent polymer.

The nanocapsules provided efficient drug release and remained stable during storage. Therefore, they could be used in many monitoring applications, including those involving the interaction of nanocapsules with target and non-target organisms. These systems may be used in different areas such as health, biotechnology, and agriculture, amongst others. Further work will be needed in order to characterize the interactions of the nanocapsules with model plants, as well as to evaluate their toxicity.

\section{Acknowledgment}

The authors are grateful for the support provided by the São Paulo Science Foundation, Brazil (FAPESP, grants \#2014/202734, \#2014/20286-9, \#2013-12322-2 and 2015/15617-9), Mexican National Council for Science and Technology (CONACYT, México) for the financial support through the project 229761/INFR-2014-02 that allowed the acquirement of the Flash photolysis equipment. Also the authors would like to thanks the bilateral cooperation project between Brazil-Mexico, join Cooperation CONACYT-CNPq (Project:174806) as well we gratefully acknowledge CONACYT's support to the present work through project 270878, Laboratorio Nacional de Materiales Grafénicos.

\section{Appendix A. Supplementary data}

Supplementary data associated with this article can be found, in the online version, at http://dx.doi.org/10.1016/j.colsurfb.2016.08 031.

\section{References}

[1] S.S. Mukhopadhyay, Nanotechnology in agriculture: prospect and constraints, Nanotechnol. Sci. Appl. 7 (2014) 63-71.

[2] M. Kah, Nanopesticides and nanofertilizers: emerging contaminants or opportunities for risk mitigation, Front. Chem. 3 (2015) 1-5.

[3] B.P. Sekhon, Nanotechnology in agri-food production: an overview, Nanotechnol. Sci. Appl. 7 (2014) 31-53.

[4] C. Parisi, M. Vigani, E. Rodriguez-Cerezo, Agricultural nanotechnologies: what are the current possibilites? Nanotoday 10 (2015) 124-127.

[5] E.V.R. Campos, J.L. Oliveira, L.F. Fraceto, Applications of controlled release system for fungicides, herbicides, acaricides, nutrients, and plant growth, hormones: a review, Adv. Sci. Eng. Med. 6 (2014) 1-15.

[6] E.V.R. Campos, J.L. Oliveira, L.F. Fraceto, B. Singh, Polysaccharides as safer release system for agrochemicals, Agron. Sustain. Dev. 35 (2015) 47-66.

[7] S.J. Klaine, P.J.J. Alvarez, G.E. Batley, T.F. Fernades, R.D. Handy, D.Y. Lyon, S. Mahendra, M.J. McLaughlin, J.R. Lead, Nanomaterials in the environment: behavior, fate, bioavailability, and effects, Environ. Toxicol. Chem. 27 (2008) 1825-1851.

[8] M.A. Maurer-Jones, I.L. Gunsolus, C.J. Murphy, C.L. Haynes, Toxicity of engineered nanoparticles in the environment, Anal. Chem 85 (2013) 3036-3049.

[9] K.L. Garner, A.A. Keller, Emerging patterns for engineered nanomaterials in the environment: a review of fate and toxicity studies, J. Nanopart. Res. 16 (2014) 2503.

[10] M. Sajid, M. Ilyas, C. Basheer, M. Tariq, M. Daud, N. Baig, F. Shehzad, Impact of nanoparticles on human and environment: review of toxicity factors, exposures, control strategies, and future prospects, Environ. Sci. Pollut. Res. 22 (2015) 4122-4143.

[11] L. Shang, K. Nienhaus, G.U. Nienhaus, Engineered nanoparticles interacting with cells: size matters, J. Nanobiotechnology. 12 (2014) 1-11. 
[12] C. Simonsson, G. Bastiat, M. Pitorre, A.S. Klymchenko, J. Béjaud, Y. Mély, J.-P. Benoit, Inter-nanocarrier and nanocarrier-to-cell transfer assays demonstrate the risk of an immediate unloading of dye from stained lipid nanocapsules, Eur. J. Pharm. Biopharm. 98 (2016) 47-56.

[13] M.-H. Nguyen, J.-S. Lee, I.-C. Hwang, H.-J. Park, Evaluation of the penetration of nanocarriers into red pepper leaf using confocal laser scanning microscopy, Crop Prot. 66 (2014) 61-66.

[14] J. Yan, M.C. Estévez, J.E. Smith, K. Wang, X. He, L. Wang, W. Tan, Dye-doped nanoparticles for bioanalysis, Nanotoday 3 (2007) 44-50.

[15] A.B. Chinen, C.M. Guan, J.R. Ferrer, S.N. Barnaby, T.J. Merkel, C.A. Mirkin, Nanoparticles probes for the detection of cancer biomarkers, cells, and tissues by fluorescence, Chem. Rev. 115 (2015) 10530-10574.

[16] O.S. Wolfbeis, An overview of nanoparticles commonly used in fluorescent bioimaging, Chem. Soc. Rev. 44 (2015) 4743-4768.

[17] G. Castruita, E. Arias, I. Moggio, F. Pérez, D. Medellín, R. Torres, R. Ziolo, A Olivas, E. Giorgetti, M. Muniz-Miranda, Synthesis, optical properties and supramolecular order of $\pi$-conjugated 25 -di(alcoxy) phenyleneethynylene oligomers, J. Mol. Struct. 936 (2009) 177-186.

[18] T. Maillou, J. Le Moigne, V. Dumarcher, L. Rocha, B. Geffroy, J.-M. Nunzi, Oligo (phenylethynylene) as a high photoluminescence quantum yield material and its distributed feedback laser emission in thin films, Adv. Mat. 14 (2002) 1297-1301.

[19] R.H. Friend, R.W. Gymer, A.B. Holmes, J.H. Burroughes, R.N. Marks, C. Taliani, D.D.C. Bradley, D.A. dos Santos, J.L. Brédas, M. Lögdlund, W.R. Salaneck, Electroluminescence in conjugated polymer, Nature 397 (1999) 121-128.

[20] P. Karam, A.A. Hariri, C.F. Calver, X. Zhao, K.S. Schanze, G. Cosa, Interaction of anionic phenyleneethylene polymers with lipids: from membrane embedding to liposome fusion, Langmuir. 30 (2014) 10704-10711.

[21] M.H. Pérez, J. Siepmann, C. Zinutti, A. Lamprecht, N. Ubrich, M. Hoffman, R. Bodmeier, P. Maincent, Non-degradable microparticles containing a hydrophilic and/or a lipophilic drug: preparation, characterization and drug release modeling, J. Control Release. 88 (2004) 413-428.

[22] E. Marin, M.I. Briceno, C. Caballero-George, Critical evalutaion of biodegradable polymers used in nanodrugs, Int. J. Nanodrugs. 8 (2013) 3071-3091.

[23] A.T.R. Williams, S.A. Winfield, J.N. Miller, Relative fluorescence quantum yields using a computer-controlled luminescence spectrometer, Analyst 108 (1983) 1067-1071.

[24] Z. Zhou, J. Ye, L. Chen, A. Ma, F. Zou, Simultaneous determination of ropivacaine and dexamethasone in biodegradable PLGA microspheres by HPLC, Yakugaku Zasshi. 130 (2010) 1061-1068.

[25] J.L. Oliveira, E.V.R. Campos, C.M.G. Silva, T. Pasquoto, R. Lima, L.F. Fraceto, Solid lipid nanoparticles co-loaded with simazine and atrazine: preparation, characterization, and evaluation of herbicidal activity, J. Agric. Food Chem. 63 (2015) 422-432.

[26] J. Asrar, Y. Ding, R.E. La Monica, L.C. Ness, Controlled release of tebuconazole from a polymer matrix microparticle: release kinetics and length of efficacy, J. Agric. Food Chem. 52 (2004) 4814-4820.

[27] E.V.R. Campos, J.L. Oliveira, C.M.G. da Silva, M. Pascoli, T. Pasquoto, R. Lima, P.C. Abhilash, L.F. Fraceto, Polymeric and solid lipid nanoparticles for sustained release of carbendazim and tebuconazole in agricultural applications, Sci. Rep. 5 (2015) 13089.

[28] D. Meza, E. Arias, I. Moggio, J. Romero, J.M. Mata, R.M. Jimenez-Barrera, R.F. Ziolo, O. Rodríguez, M. Ottonelli, Synthesis and photophysical and supramolecular study of $\pi$-conjugated (diethylene glycol methyl ether), Polym. Chem. 6 (2015) 1639-1648.

[29] V.C.F. Mosqueira, P. Legrand, H. Pinto-Alphandary, F. Puisieux, G. Barrat, Poly(d,l-lactude) nanocapsules prepared by a solvente displacement process: influence of the composition on physicochemical and structural properties, J. Pharm. Sci. 89 (2000) 414-626. 